\title{
Pre-Darcy Flow Revisited under Experimental Investigation
}

Fahd Siddiqui*, Mohamed Y Soliman, Waylon House and Akif Ibragimov

Texas Tech University, 2500 Broadway, Lubbock TX, 79409, USA

\begin{abstract}
Sufficient literature has been published about Pre-Darcy flow in non-petroleum disciplines. Investigators dissent about the significance of deviation of Darcy's Law at very low fluid velocities. Most of their investigations are based on coarse, unconsolidated porous media with an aqueous fluid. However little has been published regarding the same for consolidated oil and gas reservoirs. If a significant departure from Darcy's Law is observed, then this could have multiple implications on: reservoir limit tests, under prediction of reserves, unrecognized prospecting opportunities etc. This study aims to perform a comprehensive review of the literature; and to experimentally demonstrate that the Pre-Darcy flow effect is significant in petroleum rocks.
\end{abstract}

Keywords: Pre-Darcy flow; Non-Darcy flow; Fluid flow through porous media

\section{Nomenclature}

A Cross-sectional area, $\mathrm{m}^{2}$

a Empirical parameter

$\mathrm{h} \quad$ Formation thickness, $\mathrm{m}$

k Permeability, $\mathrm{m}^{2}$

$\mathrm{Kv} \quad$ Modified Bessel function of the second kind of order $\mathrm{v}$

m Empirical parameter

$\mathrm{n} \quad$ Flow behavior index (power law parameter)

$\mathrm{p}$ Pressure, $\mathrm{Pa}$

Q Flow rate, $\mathrm{m}^{3} / \mathrm{s}$

v Superficial velocity, $\mathrm{m} / \mathrm{s}$

$\mathrm{x} \quad$ Distance, $\mathrm{m}$

$\mathrm{z} \quad$ Laplace parameter

$\mu \quad$ Viscosity, Pa.s

$\mu$ eff Effective viscosity, Pa.sn.m1-n

\section{Introduction}

Modern petroleum engineers have used many equations to describe the physics behind the fluid flow through porous media. Under ideal situations these equations, which form the basis of modern software, yield accurate results. However, ever so often engineers are faced with challenging problems that seemingly defy physics: be it a well test problem, a history matched simulation model, or even a tool as simple as the material balance. Upon further investigation, engineers have to concede to the simple explanation that the assumptions behind those equations were violated. Even further discomforting is the admission that engineers have not yet properly characterized the physics behind the fluid flow through porous media.

Darcy's pioneering work is at the heart of all equations related to porous media. Often engineers use it without question. Forchheimer [1] demonstrated the departure from linearity for high velocity flows. However little has been said about the validity of Darcy Law at low velocities. Considerable amount of work [2-4] has already been published in this area outside of petroleum, but it has not seeped through the petroleum engineering literature.

\section{Darcy's law}

Darcy's Law is based on the experimental observation that the apparent fluid velocity is proportional to the applied pressure gradient on a porous medium. This observation is analogous to flow of fluid through pipes, capillaries (Poisseuille's Law) and also to that of flow of current through a resistive conductor. Wyckoff, [5] separated Darcy's original constant of proportionality into permeability (a property of the porous medium) and viscosity (a property of the fluid). The final form of equation is (Equation 1):

$$
v=\frac{Q}{A}=-\frac{k}{\mu} \frac{d p}{d x}
$$

Following sections discuss some of the well-known departures from Darcy's Law.

Post Darcy flow effect: Forchheimer [1] made observations that the Darcy's Law deviated from linearity for high velocities. $\mathrm{He}$ attributed this to the inertial losses. He proposed a velocity squared term to account for this non-linearity. Even in 1901, Forchheimer noted that some experimental data does not fit his newly proposed quadratic flow equation. He then proposed the addition of a cubic term to describe those data. Due to the less than proportional increase in flow velocity with respect to applied pressure gradient, this effect has shown a significant influence on well performance [6,7]. This effect is generally termed as Non-Darcy flow; however in this study we will refer to it as Post-Darcy flow. Later studies [8-10] have published the impact of Post-Darcy flow on fractured gas wells. The literature already has effectively dealt with Post-Darcy flow and the reader is suggested to consult elsewhere for a more comprehensive treatment of the subject.

*Corresponding author: Fahd Siddiqui, Texas Tech University, 2500 Broadway, Lubbock TX, 79409, USA, Tel: +1 806-742-2011; E-mail: fahdsiddiqui@gmail.com

Received April 21, 2015; Accepted October 15, 2015; Published October 25 2015

Citation: Siddiqui F, Soliman MY, House W, Ibragimov A (2015) Pre-Darcy Flow Revisited under Experimental Investigation. J Pet Environ Biotechnol 6: 249. doi:10.4172/2157-7463.1000249

Copyright: (c) 2015 Siddiqui F, et al. This is an open-access article distributed under the terms of the Creative Commons Attribution License, which permits unrestricted use, distribution, and reproduction in any medium, provided the original author and source are credited. 
Low pressure Klinkenberg effect: This effect is also well known and ascribed to the Knudsen effect (slippage effect). Also known as the Klinkenberg effect [11], who demonstrated that the permeability of a porous medium is a function of gas pressure. Well known published procedures exist to deal with this effect.

Non-newtonian fluid effect: Darcy's Law does not apply to nonNewtonian fluid flow. For non-Newtonian fluids, viscosity is a function of applied shear rate. Bird, Stewart, and Lightfoot [12] and Savins [13] gave an analogous expression of Darcy's Law for Power Law fluids (Equation 2).

$$
v^{n}=-\frac{k}{\mu_{\text {eff }}} \frac{d p}{d x}
$$

Siddiqui [14] applied the above equation and solved the radial diffusivity equation for analyzing pressure transient tests. They validated the above equation with real field injection data.

\section{Pre-Darcy flow}

Oil and gas flowing at very low velocity will be referred to as PreDarcy flow in this text. Many authors [15] have already pointed out the necessity of considering the Pre-Darcy flow. The actual fluid flow velocity in a real reservoir is very slow especially for radial flow. The Darcy velocity is superficial velocity and it can be easily related to the continuity equation by (Equation 3).

$$
v=\frac{Q}{A}
$$

In radial flow, the cross-sectional area to flow increases, which causes a decrease in fluid velocity for any, given constant flow rate.

Figure 1 shows a plot of typical oil/gas wells under steady state radial flow regime for 160 acre spacing. Figure 1 shows that at smaller radial distances, the velocity is large, but it rapidly drops to small values for intermediate to large radial distances. Another important conclusion from Figure 1 is that only for the initial 5 to $10 \%$ of the radial distance, the fluid is flowing with a high velocity; whereas more than $90 \%$ of the fluid in the porous medium is actually moving with a very small velocity (orders of magnitude smaller). Therefore Pre-Darcy is the dominant flow phenomenon in typical radial flow regime wells. Hence it warrants an experimental investigation and mathematical modeling (Figure 1).

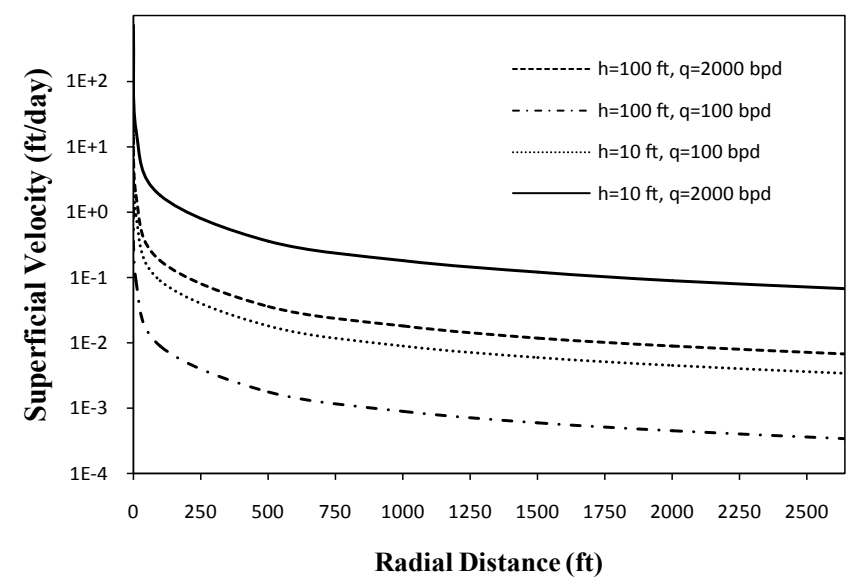

Figure 1: Superficial Velocity vs. Radial Distance for Various Flow Rates and Net-pay Thicknesses.

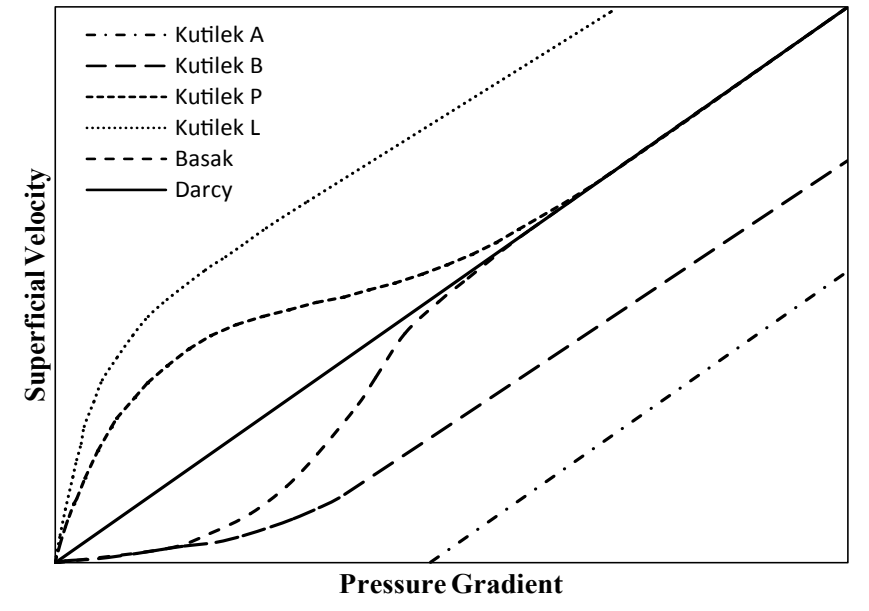

Figure 2: Pre-Darcy Regime Models and Classification [20].

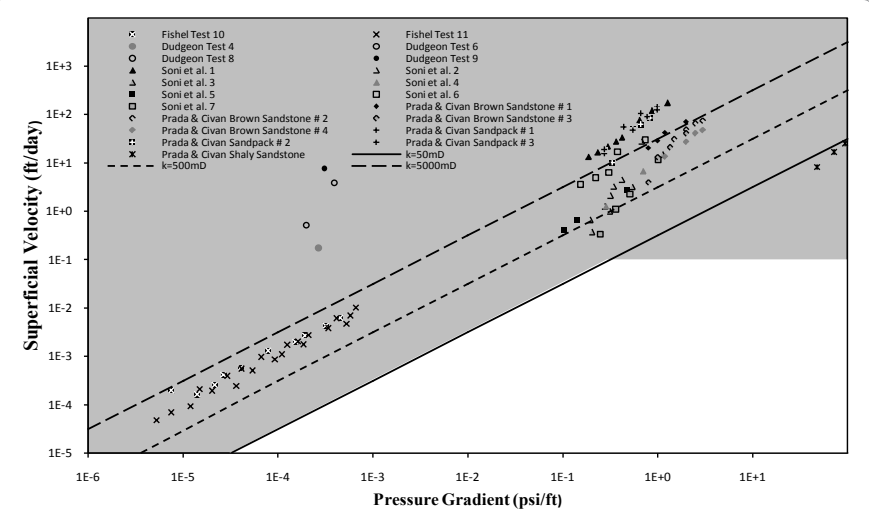

Figure 3: Published Datasets Compared with Real Reservoirs.

Researchers [16-19] over the years have also realized that not only is Pre-Darcy a deviation from linearity, but there is also a presence of a "threshold pressure gradient" and that Darcy's Law should be corrected for that. Figure 2 summarizes some of the proposed models for PreDarcy flow in the literature (with and without the threshold gradient). Kutilek [20] classified these regimes into seven types, four of which are shown in Figure 2.

Various non-Petroleum engineering literature [2-4] have already demonstrated deviations from Darcy linearity under very small velocity fluid flow. However most of those studies were conducted on unconsolidated samples. Following sections examine their experiments and conclusions (Figure 2).

\section{Published Datasets}

Figure 3 summarizes some of the datasets collected for Pre-Darcy flow. It also yields a comparison of real reservoirs velocities versus the lab experiments.

Fishel [2] observed that laboratory tests for permeability are made with much higher pressure gradients than those encountered in water bearing formations. He conducted experiments with sand samples and water as the working fluid in a U-tube apparatus. His conclusions were that Darcy's Law is valid for very low velocities $\left(10^{-4} \mathrm{ft} /\right.$ day and above)

Dudgeon [3] conducted permeability tests on coarse grained 
material including river gravels, crushed rock particles and glass marbles with water as the working fluid. He was able to discern three different regimes from his experiments: Pre-Linear (Pre-Darcy), Linear (Darcy) and Post-Linear (Post-Darcy). These flow regimes are distinguishable with abrupt changes in linearity. Therefore Dudgeon proposed an empirical fit based on Equation 4 [21-23]. Where the coefficients $a$ and $m$ are different for each flow regime and that $m=1$ for Darcy flow and $m<1$ for Pre-Darcy flow. Dudgeon's explanation for the Pre-Linear flow was ascribed to non-Newtonian characteristics caused by interfacial tension.

$$
\frac{\partial p}{\partial x}=a v^{m}
$$

Soni et al. [4] conducted various experiments on different particle sized porous media. The objective of their study was to better correlate the values of $a$ and $m$ for particle size and porosities. Their experiments also suggested abrupt changes in flow regimes and categorized them into Pre-Linear, Linear and Post Linear flow regimes; and they too concluded that $m<1$ for Pre-Darcy flow. Their experiments were conducted with particle sizes in the range of 0.074 to $1.19 \mathrm{~mm}$ and with porosities as high as $48.75 \%$. They were able to identify Pre-Darcy regime for velocity as high as $100 \mathrm{ft} /$ day. This kind of information is relevant to unconsolidated reservoirs and shows qualitatively that even the near wellbore region might be experiencing Pre-Darcy flow phenomenon.

Prada and Civan [19] experimentally demonstrated the existence of a threshold pressure gradient for liquids. They attributed this threshold to frictional effects. Their experiments were conducted on consolidated sandstones, sand-packs and on shaly sandstone; with brine as the working fluid. They demonstrated that the threshold pressure gradient is an inverse power law of mobility (Figure 3).

Neuzil [24] attributed the departures from Darcy's law in the Pre-Darcy range to subtle experimental errors: changes in water viscosity, measurement errors, small leaks, bacterial activity, incorrect assumption of steady state flow, gas generation and dissolution, and changes in medium matrix. However he also conceded that an observational gap exists, and that flow measurements have only been made at gradients several orders of magnitudes higher than in actual nature. Therefore applicability of Darcy's Law can only be inferred at small gradients.

Figure 3 also shows the velocity vs. gradient lines for different permeabilities encountered in petroleum reservoirs. These lines show that almost all of the experiments conducted were on high permeability $(\mathrm{k}>500 \mathrm{mD})$ media and also confirm that most of the experimental data is not parallel to these lines (hence under Pre-Darcy flow as discussed above). The shaded region shows the reservoirs with a permeability of $50 \mathrm{mD}$ or less. Inspection of Figure 3 alongside Figure 1 reveals that only the experiments conducted [2] were in the low velocity range $(v<0.1 \mathrm{ft} /$ day $)$. As described earlier, at least $80 \%$ of the porous media is flowing fluid with the velocity of $0.1 \mathrm{ft} /$ day or lower.

Noting that most petroleum reservoirs have a permeability of 50 $\mathrm{mD}$ or less and that $80 \%$ of the fluid in a typical reservoir is flowing with a velocity of $0.1 \mathrm{ft} /$ day or less; a "region of interest" can be constructed on Figure 3 (shaded) based on these constraints. This region describes a real petroleum reservoir having a permeability of $50 \mathrm{mD}$ (or less) dominated with low velocity flow ( $0.1 \mathrm{ft} /$ day or less). It becomes apparent that none of the experiments were conducted in this region of interest.

\section{Experimental Work}

Most of the published work was concerned with coarse unconsolidated material with water as the working fluid. In this study, authors carried out experiments on consolidated porous media with an organic (Soltrol-130) fluid. This study employed the U-tube apparatus described by Fishel [2], to apply low pressure gradients on the medium. This study aims to target the "region of interest" (as shaded on Figure 3).

\section{Experimental setup}

The U-tube type apparatus (Figure 3) applies the pressure gradient on a saturated core sample through the difference of fluid levels in the columns. This difference in head is then converted to pressure difference through the density of the working fluid. This pressure difference is converted to pressure gradient by dividing by the length of the core sample. The flow rate is calculated by noting the change in head with respect to time. By using the diameter of the tube (crosssectional area) this rate of head change is converted to rate of volume change (flow rate). Superficial velocity can be found by dividing the flow rate by the cross-sectional area of the core sample (Equation 3 ).

Consolidated core samples from sandstone reservoirs of various permeabilities were used to study the Pre-Darcy effect in these experiments (Figure 4 ).

The required data of head vs. time were recorded for core samples with various lengths, diameters and permeabilities. Velocity vs. gradient data (after calculations) is plotted on Figure 5.

\section{Laboratory precision and uncertainty}

The main sources of error in the experiment arise from the challenge of measurement of low flow rates and low pressure gradients. The setup described above can read pressures down to $1 \mathrm{~mm}$ head $(9.8$ $\mathrm{Pa}$ or $1.4 \times 10^{-3} \mathrm{psi}$, assuming water head, Soltrol-130 would result in even smaller least count). Low flow rates can also be directly read off down to zero with this setup.

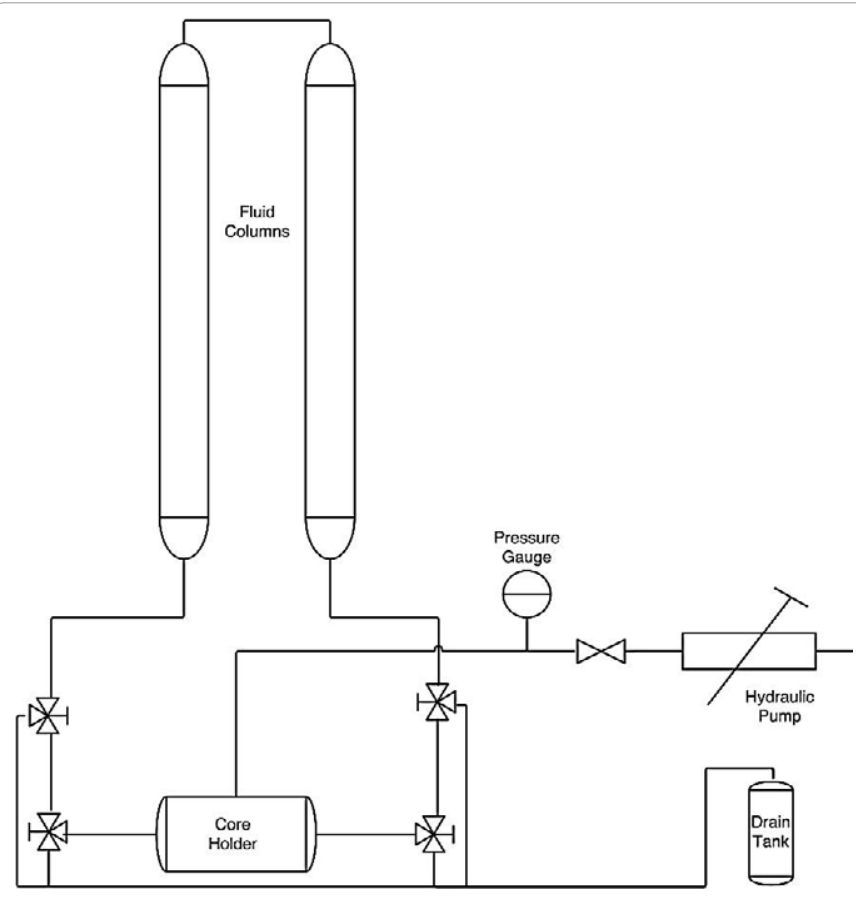

Figure 4: Schematic of the U-tube Type Apparatus. 


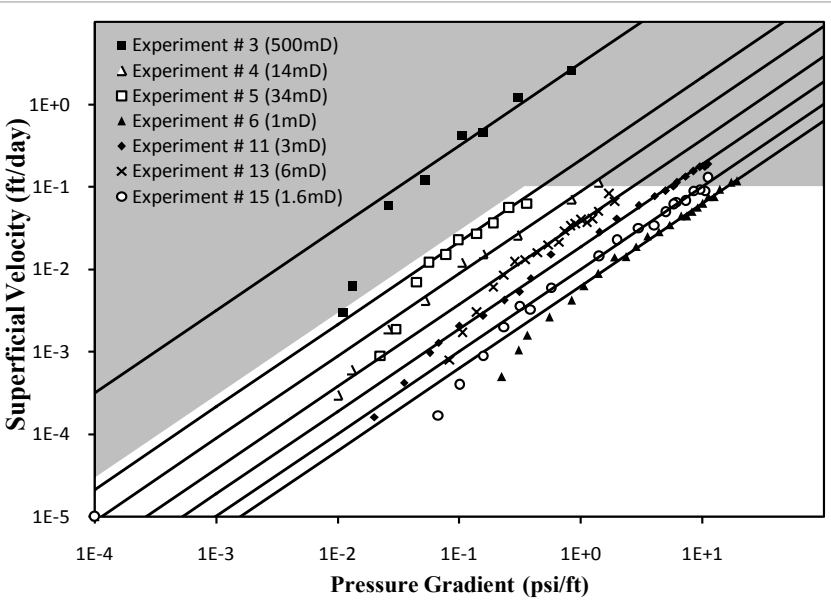

Figure 5: Experimental Results Targeting the "Region of Interest".

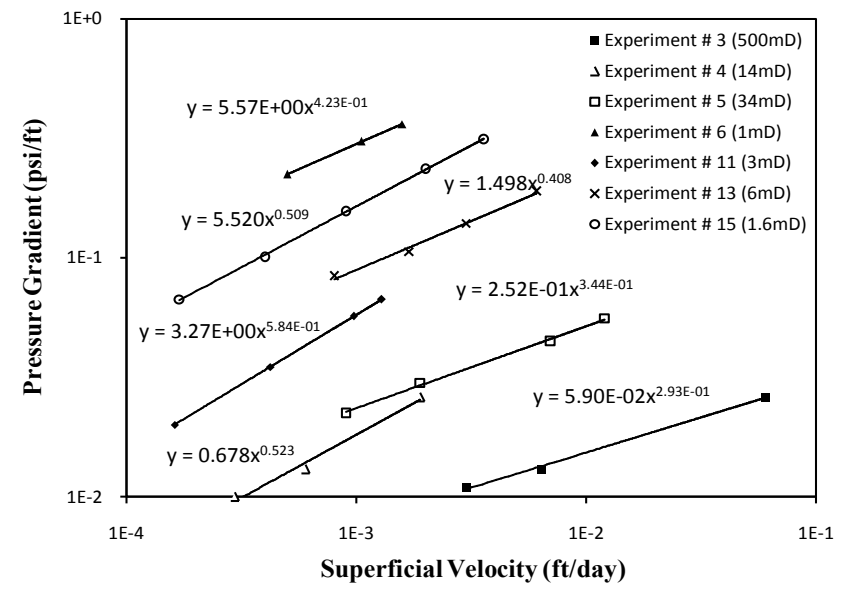

Figure 6: Power Law Applied to Pre-Darcy Regime.

Constant temperature was achieved using the laboratory HVAC system to maintain the temperature at $19^{\circ} \mathrm{C}$. The temperature variations were monitored every half hour and found to be within $0.5^{\circ} \mathrm{C}$. The sample was cleaned, dried and vacuumed before saturating with Soltrol-130 to remove impurities and air from the system. Constant confining pressure was applied with a hydraulic pump on the core holder to avoid annular flow and wall effects. The fluid columns were connected at the top to avoid evaporation loss (which may contribute to misleading results), while at the same time maintaining atmospheric pressure above the liquid level. Material balance checks were performed to establish certainty and accuracy of data.

These precautions helped with taking precise readings to avoid the experimental errors suspected by Neuzil [24]. Moreover since the authors used organic fluid instead of water, this study avoids the electrostatic effects associated with the polar nature of water [25].

\section{Results}

Experimental data and results are plotted on Figure 5 (which is an extension of Figure 3). The experiments were conducted on samples with a range of permeability targeting the "region of interest" (shaded). It becomes evident that the Pre-Darcy phenomenon occurs abruptly (as pointed out by other researchers) below a velocity value that depends on the medium. At these velocities, the plot of superficial velocity

\begin{tabular}{|c|c|c|c|}
\hline 1 & $\mathrm{ft} /$ day & $3.528 \mathrm{E}-06$ & $\mathrm{~m} / \mathrm{s}$ \\
\hline 1 & $\mathrm{stb} /$ day & $1.840 \mathrm{E}-06$ & $\mathrm{~m}^{3} / \mathrm{s}$ \\
\hline 1 & $\mathrm{psi}$ & 6895 & $\mathrm{~Pa}$ \\
\hline 1 & $\mathrm{ft}$ & 0.3048 & $\mathrm{~m}$ \\
\hline 1 & $\mathrm{mD}$ & $9.872 \mathrm{E}-16$ & $\mathrm{~m}^{2}$ \\
\hline 1 & $\mathrm{psi} / \mathrm{ft}$ & 22621 & $\mathrm{~Pa} / \mathrm{m}$ \\
\hline
\end{tabular}

Table 1: Unit Conversions.

versus pressure gradient deviates from linear relationship.

Figure 6 shows the power law fit (described by Equation 4) on the Pre-Darcy regime of the experimental results. The value of $m$ was found to vary between 0.3 and 0.6 for these particular experiments. This shows significant Pre-Darcy effect at these velocities. However $m$ does not correlate well with the permeability and therefore $m$ can only be determined experimentally. Clearly since $a$ is a function of permeability our experiments also show that $a$ varies inversely with permeability (Figure 5).

Deviation from unit slope also points towards the existence of a "threshold pressure gradient" as described by previous researchers. However for this study it was considered that Equation 4 is sufficient to describe mathematically the physics behind the Pre-Darcy phenomenon. However our experimental data does corroborate the existence of a threshold pressure gradient (Figure 6).

\section{Conclusion}

1. The literature shows surprising lack of low velocity experiments on petroleum rocks.

2. Pre-Darcy effect is relevant because real field reservoirs are dominated by Pre-Darcy (low velocity) flow.

3. Experiments from previous non-petroleum literature are inconclusive about the existence of significant Pre-Darcy effect.

4. Those experiments, that confirm the existence of a PreDarcy effect, were conducted on coarse unconsolidated material with an aqueous fluid; which casts a shadow on applicability to petroleum reservoirs.

5. A "region of interest" (to petroleum engineers) was identified and none of the published experiments were conducted in that region.

6. This study experimentally showed the existence of Pre-Darcy effects on consolidated core samples with organic fluid (Table 1).

\section{References}

1. Forchheimer $P$ (1901) Wasserbewegung durch Boden. Zeits. V Deutsch Ing 45: 1781.

2. Fishel VC (1935) Further tests of permeability with low hydraulic gradients Transactions of the American Geophysical Union 16: 499-503.

3. Dudgeon CR (1966) An Experimental Study of the Flow of Water Through Coarse Granular Media. La Houille Blanche 7: 785-801.

4. Soni JP, Islam N, Basak P (1978) An Experimental Evaluation of Non-Darcian Flow in Porous Media. Journal of Hydrology 38: 231-241.

5. Wyckoff RD, Botset HG, Muskat M, Reed RW (1933) The Measurement of Permeability of Porous Media for Homogeneous Fluids. Review of Scientific Instruments 4: 394-405.

6. Firoozabadi A, Katz DL (1979) An analysis of high-velocity gas flow through porous media. Journal of Petroleum Technology 211-216.

7. Evans RD, Civan F (1994) Characterization of Non-Darcy Multiphase Flow in Petroleum Bearing Formation. Bartlesville, OK, US Department of Energy USA. 
Citation: Siddiqui F, Soliman MY, House W, Ibragimov A (2015) Pre-Darcy Flow Revisited under Experimental Investigation. J Pet Environ Biotechnol 6: 249. doi:10.4172/2157-7463.1000249

Page 5 of 5

8. Holditch SA, Morse RA (1976) The effects of non-Darcy flow on the behavior of hydraulically fractured gas wells. Journal of Petroleum Technology 28: 11691196.

9. Guppy KH, Cinco-Ley H, Ramey HJ (1982) Pressure buildup analysis of fractured wells producing at high flowrates. Journal of Petroleum Technology 34: $2656-2666$

10. Martins JP, Milton-Taylor D, Leung HK (1990) The effects of non-Darcy flow in propped hydraulic fractures. Proceedings of the SPE Annual Technical Conference (p. SPE 20790). New Orleans, Louisiana: SPE.

11. Klinkenberg LJ (1941) The Permeability of Porous Media to Liquids and Gases American Petroleum Institute, Drilling and Production Practice, 200

12. Bird RB, Stewart WE, Lightfoot EN (1960) Transport Phenomena. New York City: John Wiley and Sons Inc.

13. Savins JG (1969) Non-Newtonian Flow Through Porous Media. Industrial and Engineering Chemistry 61: 18-47.

14. Siddiqui F, Soliman MY, House W (2014) A New Methodology for Analyzing Non-Newtonian Fluid Flow Tests. Journal of Petroleum Science and Engineering 124: 173-179.

15. Longmuir G (2004) Pre-Darcy Flow: A Missing Piece of the Improved Oil Recovery Puzzle? SPE/DOE Fourteenth Symposium on Improved Oil Recovery $17-21$
16. Hubbert MK (1956) Darcy's Law and two field equations of the flow of underground fluids. Petroleum Transactions AIME 222-239.

17. Tek MR (1957) Development of a Generalized Darcy Equation. Journal of Petroleum Technology 9: 45-47.

18. Das AK (1997) Generalized Dary's Law including source effect. Journal of Canadian Petroleum Technology 36: 57-59.

19. Prada A, Civan F (1999) Modification of Darcy's law for the threshold pressure gradient. Journal of Petroleum Science and Engineering 22: 237-240.

20. Kutilek M (1972) Non-Darcian Flow of Water in Soils - Laminar Region: A Review. Fundamentals of Transport Phenomena in Porous Media 327.

21. Escande L (1953) Experiments concerning the filtration of water through a rock mass. Reprint from Proceedings Minnesota International Hydraulics Convention 547-553.

22. Slepicka $F$ (1961) The Laws of filtration and limits of their validity. I.A.H.R Proceedings 9th Convention 38: 3-394.

23. Anandakrishan M, Varadarajulu GH (1963) Laminar and turbulent flow of water through sand. A.S.C.E. Proceedings 89: 1-16.

24. Neuzil CE (1986) Groundwater Flow in Low-Permeability Environments. Water Resources Research 22: 1163-1195.

25. Basak P (1977) Non-Darcy Flow and Its Implications to Seepage Problems Journal of the Irrigation and Drainage Division 103: 459-473. 